\title{
Alterações miofuncionais orofaciais associadas ao processo de envelhecimento em um grupo de idosos institucionalizados
}

\author{
Sheila Tamanini de Almeida*, Bruna Caon Gentil**, Eveline de Lima Nunes ${ }^{* *}$
}

\section{Resumo}

Com o aumento da população idosa, os profissionais da saúde devem estar preparados para atuar com essa faixa etária, uma vez que ocorrem mudanças fisiológicas e estruturais em consequência do envelhecimento. O objetivo do presente estudo foi verificar as consequências que as alterações miofuncionais orofaciais causam nas funções estomatognáticas de mastigação e de deglutição em idosos institucionalizados. O estudo foi realizado na Sociedade Porto-Alegrense de Auxílio aos Necessitados (Spaan), sendo avaliados 15 idosos entre 64 e 93 anos, com queixa para mastigar e/ou deglutir. Foi realizada entrevista sobre as possíveis dificuldades no processo de alimentação e, após, foram aplicados protocolos para avaliação das estruturas estomatognáticas e das funções de mastigação e de deglutição. Verificou-se que 8 $(53,33 \%)$ idosos entrevistados fazem uso de prótese dentária; 6 (40\%) apresentaram mastigação bilateral alternada; 4 (26,67\%), mastigação unilateral direita; e 6 (40\%), mastigação com participação exagerada da musculatura perioral. A mastigação lenta foi observada em 13 (86,67\%) dos avaliados, e a movimentação vertical de mandíbula, em 6 (40\%). Verificou-se deglutição com alteração em 4 (26,67\%) idosos, dos quais $2(13,33 \%)$ apresentaram engasgo ao deglutir; um com $(6,67 \%)$ presença de tosse e $1(6,67 \%)$ demonstrando deglutição ruidosa. Não foi encontrada significância entre as alterações de mastigação e de deglutição e o desempenho nesses processos, mesmo havendo queixa na realização das funções e alteração nas estruturas estomatognáticas.

Palavras-chave: Envelhecimento. Saúde do idoso. Mastigação. Prótese dentária. Sistema estomatognático.

* Fonoaudióloga. Professora Assistente do Departamento de Fonoaudiologia da Universidade Federal de Ciências da Saúde de Porto Alegre. Especialista em Motricidade Orofacial e Disfagia. Mestra em Ciências da Saúde pela UFRGS. Endereço para correspondência: Departamento de Fonoaudiologia. Rua Sarmento Leite, 245, CEP: 90050-170, Porto Alegre - RS. E-mail: coordfono@ufcspa.edu.br.

** Fonoaudióloga. Graduanda do curso de Análise de Políticas e Sistemas de Saúde na Universidade Federal do Rio Grande do Sul.

**** Fonoaudióloga. Aluna da Pós-Graduação em Fonoaudiologia Hospitalar da Universidade Veiga de Almeida.

$\hookrightarrow$ doi:10.5335/rbceh.2012.026 


\section{Introdução}

$\mathrm{Na}$ atualidade do Brasil, observa-se o aumento da população idosa, uma vez que há diminuição da natalidade e da mortalidade, concomitantemente ao aumento da expectativa de vida. (BIAZUZ; BALBINOT; WIBELINGER, 2010). Segundo o Censo de 2010 do Instituto Brasileiro de Geografia e Estatística (IBGE), $15 \%$ da população total de Porto Alegre têm 60 anos ou mais. Dessa forma e uma vez que a melhoria da qualidade de vida do idoso tem relação direta com a qualidade do atendimento recebido e com o desenvolvimento e a aplicação da pesquisa na área da saúde (SILVA; GOLDENBERG, 2001; LOCKER et al., 2002), os profissionais que nela atuam devem estar atentos às modificações estruturais e fisiológicas provocadas pelo envelhecimento nessa população. (MENDES; TCHAKMAKIAN, 2009).

As modificações causadas pelo envelhecimento podem afetar o sistema estomatognático (SEG) (BIANCHINI, 2002; KOSSIONI; DONTAS, 2007), composto por músculos, ossos, dentes, articulação temporomandibular, sistema vascular, sistema nervoso e espaços vazios. $O$ SEG desempenha as funções de sucção, mastigação, deglutição, respiração e fala, podendo qualquer alteração que afete suas estruturas acarretar, além da mudança morfológica, distúrbios em suas funções. (ALTMANN, 1990; MARCHESAN, 1997).

Estudos realizados com idosos demonstram a ocorrência da diminuição da massa, do volume e do número de fibras musculares, afetando a força muscular, e, também, da diminuição do controle dos movimentos, deixando a musculatura menos elástica e menos flexível. Esses fatores podem explicar as alterações na tonicidade e na mobilidade dos órgãos fonoarticulatórios. (MOJON; BUDTZ-JORGENSEN; RAPIN, 1999; SASAHARA; LIMA, 2001; FAZITO et al., 2004).

As modificações presentes no processo de envelhecimento causam grande dificuldade para o idoso ingerir alimentos sólidos, e, muitas vezes, o meio facilitador é o uso de alimentos líquidos. (DIAS; CARDOSO, 2009). Estudos comprovam que problemas alimentares estão relacionados a modificações nas funções orofaciais, à má postura e ao posicionamento das estruturas motoras orofaciais, além da ausência de dentes e do uso de próteses dentárias, comumente encontradas em residentes de instituições de longa permanência para idosos (ILPIs). (O'LOUGHLIN; SHANLEY, 1998; BOURDIOL; MIOCHE, 2000; CANTERJI et al., 2004; LIMA et al., 2004).

Nesse sentido, a fonoaudiologia vem evoluindo por meio da prática baseada em evidências na reabilitação de idosos, os quais são encaminhados para tratamento por médicos e por dentistas devido a dificuldades para deglutir, a próteses mal adaptadas, a problemas periodontais e, até mesmo, a dificuldades na fala. (SILVA; GOLDENBERG, 2001). Numa instituição asilar, o fonoaudiólogo, além de ter o papel de resgatar a linguagem do idoso, deve estar atento às condições de audição, de voz, da motricidade orofacial e, em especial, às questões relacionadas ao processo de alimentação, a fim de pro- 
mover condições para uma alimentação equilibrada e prazerosa, tendo em vista as modificações diante do envelhecimento normal e, sobretudo, do patológico.

Em estudo realizado por Silva e Goldenberg (2001), em uma ILPI, os idosos foram entrevistados quanto às queixas fonoaudiológicas. A terceira queixa mais prevalente foi relacionada ao processo de alimentação, o que reforça a importância da pesquisa e assistência nessa área das instituições.

Nesse contexto, o presente estudo tem como objetivo verificar as consequências que as alterações miofuncionais orofaciais decorrentes do processo de envelhecimento acarretam nas funções estomatognáticas de mastigação e de deglutição em um grupo de idosos institucionalizados.

\section{Metodologia}

O estudo foi realizado de abril a agosto de 2011, na Sociedade Porto-Alegrense de Auxílio aos Necessitados (Spaan), em funcionamento desde 1931. Na ocasião em que se desenvolveu a pesquisa, a entidade abrigava 125 idosos carentes acima de 60 anos e de ambos os sexos.

A pesquisa caracteriza-se por um estudo transversal, observacional e descritivo, tendo sido aprovada pelo Comitê de Ética em Pesquisa da Universidade Federal de Ciências da Saúde de Porto Alegre com registro n. 716/10.

$\mathrm{O}$ estudo foi iniciado com a coleta de dados, realizada pelas pesquisadoras com base na leitura dos prontuários, objetivando verificar as informações sobre as patologias pregressas e atuais de todos os idosos residentes na instituição.
Foram considerados como critério de inclusão os idosos sem diagnóstico psiquiátrico confirmado, sem doença neurológica, sem doença progressiva ou demencial, independentes (indivíduos com capacidade de locomoção) ou independentes em cadeira de rodas (locomoção a longas distâncias com auxílio de cadeira de rodas). Foram excluídos os indivíduos que se negaram a participar da pesquisa. Todos os idosos que satisfizeram os critérios de inclusão foram informados sobre o estudo e seus procedimentos, sendo convidados a participar. Os participantes alfabetizados assinaram o Termo de Consentimento Livre e Esclarecido (TCLE), e os não alfabetizados tiveram seu TCLE assinado pelo procurador responsável da instituição.

$\mathrm{Na}$ etapa seguinte, os idosos incluídos foram entrevistados sobre sua possível dificuldade ao mastigar e/ou deglutir os alimentos e ingerir as medicações. Foram, também, registradas informações referentes à presença de doenças respiratórias, a perda ou ganho de peso nos últimos meses, ao uso de prótese dentária, além do nível de alfabetização. Nessa fase, foram entrevistados 78 idosos, tendo 15 demonstrado queixas para mastigar e/ou deglutir.

Num terceiro momento, as pesquisadoras aplicaram os seguintes protocolos: o protocolo de avaliação miofuncional orofacial (MARCHESAN, 2003) e o protocolo de avaliação das estruturas estomatognáticas. (SUZUKI, 1997). No primeiro, constam perguntas quanto à dificuldade para mastigar, à lateralidade, à existência de ruído ou de dor durante a mastigação, à produção excessiva 
de saliva e à dificuldade na produção da saliva em relação à fala; sobre a existência de engasgos ou de tosse após a alimentação; bem como sobre $o$ ajuste da prótese dentária e sua interferência na alimentação e na fala. $\mathrm{O}$ protocolo de avaliação das estruturas estomatognáticas (SUZUKI, 1997) avalia os aspectos de postura, da morfologia, da tonicidade e da mobilidade das estruturas estomatognáticas, além do desempenho das funções de mastigação e de deglutição.

A avaliação das funções de mastigação e deglutição foi realizada em uma sala pré-organizada com os materiais necessários (cadeiras, mesa, estetoscópio, luvas, espátula, filmadora, os protocolos e alimentos), por meio da avaliação de uma alimentação padrão do estudo, ou no quarto do entrevistado, por meio da observação de uma alimentação do idoso com um pão francês, uma fruta batida na consistência pastosa e água mineral em temperatura ambiente. Essa avaliação foi efetuada durante o café da manhã ou da tarde, de acordo com os horários da instituição. Para qualificar a avaliação das funções estomatognáticas, foi gravado um vídeo de cada idoso no momento da ingestão dos alimentos. A ausculta cervical foi realizada antes, durante e depois de cada alimento ser ingerido em todas as consistências oferecidas.

Os dados foram analisados por meio de tabelas, gráficos e estatísticas descritivas. Foram utilizados o teste exato de Fisher para a verificação de associação entre as variáveis qualitativas e o teste não paramétrico Mann-Whitney para a comparação da idade com as variáveis do estudo. Para esses testes, o nível de significância máximo assumido foi de $5 \%(\mathrm{p} \leq 0,05)$, e o software utilizado para a análise estatística foi o SPSS® versão 10.0 .

\section{Resultados}

Os participantes do estudo apresentavam a função cognitiva preservada, observada em avaliação informal feita pelas pesquisadoras por meio de diálogo com eles estabelecido. A menor e a maior idade apresentadas foram 64 e 93 anos, com média de 79,5 anos e desvio padrão de 8,5. Do total da amostra, 12 eram do sexo feminino e 3 do sexo masculino, sendo $10(66,67 \%)$ independentes e 5 $(33,33 \%)$ independentes em cadeira de rodas. O tempo médio de institucionalização dos idosos na Spaan foi de 4,4 anos.

As patologias de base de maior prevalência nesse grupo foram hipertensão arterial sistêmica $(33,33 \%)$, doença pulmonar obstrutiva crônica (20\%), insuficiência cardíaca $(20 \%)$ e incontinência urinária $(6,67 \%)$. Além disso, constava nos prontuários que dois $(13,33 \%)$ idosos eram ex-tabagistas. Na entrevista inicial, dos 15 idosos com queixa para mastigação e/ou deglutição, 12 apresentaram queixa para mastigar e 3 para deglutir os alimentos.

$\mathrm{Na}$ avaliação miofuncional orofacial, em relação à queixa, 12 (80\%) responderam ter dificuldade para mastigar e $3(20 \%)$, dificuldade para deglutir. Oito $(53,33 \%)$ idosos com dificuldade para mastigar relataram ter essa queixa todas as vezes que se alimentam. Quando questionados em relação ao lado mastigatório, $5(33,33 \%)$ revelaram masti- 
gação unilateral exclusiva, 2 (13,33\%) relataram mastigação unilateral rara e $8(53,33 \%)$, mastigação bilateral. A maioria dos entrevistados, $13(86,67 \%)$, referiu nunca mastigar com a boca aberta, não apresentar ruído durante a mastigação, nem durante a fala. Não relataram dor para mastigar $10(66,67 \%)$ dos idosos da amostra. Dos entrevistados, 5 $(33,33 \%)$ nunca apresentaram engasgos $\mathrm{e}$, às vezes, apresentaram tosse após a deglutição; $4(26,67 \%)$ sempre tossiam após deglutir; e 2 (13,33\%) engasgavam-se durante a alimentação. Descreveram, ainda, dificuldade na fala $3(20 \%)$ dos idosos. Um idoso não se alimentava da consistência sólida. Dos entrevistados, $7(46,67 \%)$ destacaram nunca produzir saliva excessivamente e nunca perceber dificuldade de adaptação da prótese em relação à fala ou à alimentação.

Na avaliação da morfologia de lábios, língua e bochechas - segundo aplicação do protocolo de avaliação das estruturas estomatognáticas (SUZUKI, 1997) -, a maioria dos idosos avaliados apresentou características estruturais e de postura adequadas. $\mathrm{Na}$ avaliação da estrutura labial, 3 (20\%) apresentaram lábio superior encurtado; dois (13,33\%), fino; e 5 (33,33\%), encurtado e fino. Ainda, 3 (20\%) idosos apresentaram lábio inferior fino.

$\mathrm{Na}$ avaliação das estruturas estomatognáticas referente à questão morfológica dos dentes, da prótese dentária, do palato duro e do palato mole, pôde-se observar que 11 idosos $(73,33 \%)$ apresentaram ausência de dentes, 7 (46,67\%) não possuíam prótese dentária, $2(13,33 \%)$ faziam uso de prótese parcial na arcada superior, $4(26,67 \%)$ usavam prótese total na arcada superior e 2 $(13,33 \%)$ usavam prótese total em ambas as arcadas. Não foi avaliado o palato duro de 8 idosos, devido à utilização de prótese superior.

A Tabela 1 apresenta os resultados do protocolo de avaliação das estruturas estomatognáticas em relação à tonicidade dos lábios, da língua, do mentual e das bochechas.

Tabela 1 - Distribuição de idosos de instituição de longa permanência quanto à avaliação da tonicidade: lábios, língua, mentual e bochecha. Porto Alegre, Brasil, 2011.

\begin{tabular}{llcr}
\hline Estrutura & \multicolumn{1}{c}{ Tonicidade } & $\mathrm{n}$ & $\%$ \\
\hline Lábios & $\begin{array}{l}\text { Normal } \\
\text { Superior }\end{array}$ & 11 & 73,33 \\
& $\begin{array}{l}\text { e inferior } \\
\text { Hipofuncionantes } \\
\text { Inferior } \\
\text { Hipofucionante }\end{array}$ & 3 & 20,00 \\
& Normal & 15 & 100,00 \\
\hline Língua & Normal & 10 & 66,67 \\
\hline Bochecha & direita & 5 & 33,33 \\
\hline Bochecha & Normal & 10 & 66,67 \\
esquerda & Hipofuncionante & 5 & 33,33 \\
\hline Mentual & Normal & 8 & 53,33 \\
& Hipofuncionante & 1 & 6,67 \\
& Hiperfuncionante & 6 & 40,00 \\
\hline
\end{tabular}

Quando avaliada a mobilidade dos lábios, da língua, da mandíbula e do palato mole, pela utilização do protocolo de avaliação das estruturas estomatognáticas, observou-se alteração na mobilidade de língua em $1(6,67 \%)$ idoso pesquisado. Também, $1(6,67 \%)$ idoso apresentou mau funcionamento velofaríngeo. A 
mobilidade de lábios e a de mandíbula encontraram-se adequadas em todas as avaliações.
A Tabela 2 expõe os resultados da avaliação da mastigação com alimentos pela utilização do protocolo de avaliação das estruturas estomatognáticas.

Tabela 2 - Distribuição dos achados da avaliação da mastigação em um grupo de idosos institucionalizados, segundo o protocolo de avaliação das estruturas estomatognáticas. Porto Alegre, Brasil, 2011.

\begin{tabular}{|c|c|c|c|}
\hline Critérios & Categoria & $\mathrm{n}$ & $\%$ \\
\hline \multirow[t]{5}{*}{ Tipo } & Bilateral alternada & 6 & 40,00 \\
\hline & Bilateral alternada com predomínio esquerdo & 3 & 20,00 \\
\hline & Unilateral direita & 4 & 26,67 \\
\hline & Unilateral esquerda & 1 & 6,67 \\
\hline & Centralizada & 1 & 6,67 \\
\hline \multirow{3}{*}{ Velocidade } & Rápida & 1 & 6,67 \\
\hline & Lenta & 13 & 86,67 \\
\hline & Normal & 1 & 6,67 \\
\hline $\begin{array}{l}\text { Participação } \\
\text { da musculatura }\end{array}$ & Presente & 6 & 40,00 \\
\hline perioral & Ausente & 9 & 60,00 \\
\hline Movimento & Rotatórios & 9 & 60,00 \\
\hline Mandíbula & Verticais & 6 & 40,00 \\
\hline
\end{tabular}

A aplicação do teste exato de Fisher buscou a associação entre a queixa de dificuldade para mastigar às seguintes variáveis: tipo mastigatório, velocidade de mastigação, participação da musculatura perioral durante a mastigação, movimentos de mandíbula e posição dos lábios durante o processo de mastigação. Não foram observadas associações significativas ( $\mathrm{p} \geq 0,05)$.
Quanto à influência da utilização de prótese dentária no tipo mastigatório, na velocidade de mastigação, na participação da musculatura perioral durante a mastigação, nos movimentos de mandíbula e na posição dos lábios ao longo desse mesmo processo, não houve associações significativas entre as variáveis ( $\mathrm{p} \geq 0,05)$. A Tabela 3 demonstra a avaliação da deglutição mediante utilização do protocolo de avaliação das estruturas estomatognáticas. 
Tabela 3 - Distribuição dos achados da avaliação da deglutição em idosos institucionalizados, segundo o protocolo de avaliação das estruturas estomatognáticas. Porto Alegre, Brasil, 2011.

\begin{tabular}{llcc}
\hline Critérios & \multicolumn{1}{c}{ Categoria } & $\mathrm{n}$ & $\%$ \\
\hline \multirow{3}{*}{ Engasgo } & Normal & 11 & 73,33 \\
& Alterada & 4 & 26,67 \\
& Com engasgo & 2 & 13,33 \\
Ruído & Sem engasgo & 12 & 80,00 \\
& Tosse & 1 & 6,67 \\
Participação de outras estruturas & Com ruído & 1 & 6,67 \\
& Sem ruído & 14 & 93,33 \\
& Musculatura perioral & 2 & 13,33 \\
Ausculta cervical & Projeção da cabeça & 2 & 13,33 \\
& Sem alteração & 11 & 73,33 \\
& Normal & 15 & 100,00 \\
\hline
\end{tabular}

\section{Discussão}

O sistema estomatognático, assim como todo o corpo humano, é submetido, com o passar dos anos, a diversos fatores extrínsecos e intrínsecos que alteram suas estruturas. Dessa forma, várias modificações nas estruturas orofaciais são observadas em decorrência do envelhecimento, como perda óssea, perda da resistência e da tonicidade dos músculos, problemas na articulação temporomandibular (ATM), diminuição do volume de secreção salivar e perda dentária. (FREITAS JUNIOR et al., 2008).

Dos 15 idosos que participaram do estudo, 12 eram mulheres, visto que há predominância feminina na instituição, corroborando a literatura, que refere a presença mais significativa de idosas na maioria das ILPIs. (CHAIMOWICZ ; GRECO, 1999; DAVIM et al., 2004; GUEDES; SILVEIRA, 2004; GUTIERRES et al., 2009).

No estudo de Gutierres et al. (2009), as queixas de dificuldades na mastigação e/ou deglutição foram encontradas em $48 \%$ dos idosos pesquisados, levando os autores à conclusão de que essas alterações podem ser resultado não apenas da idade, mas também das más condições da saúde bucal. No presente estudo, não houve associação significativa entre as queixas de dificuldade para mastigar encontradas com o tipo e a velocidade mastigatória, com a participação da musculatura perioral, com os movimentos de mandíbula e com a posição dos lábios durante o processo de mastigação. Tal fato pode ser explicado pelo número reduzido de sujeitos integrantes do estudo, mesmo utilizando-se estatística adequada a amostras reduzidas.

Salienta-se que todos os pacientes avaliados apresentaram queixa inicial de dificuldade para mastigação e/ou deglutição. Em 11 (73,33\%) dos avaliados, observou-se ausência de dentes, e os demais apresentaram presença de dentes naturais, com regular conservação dentária. Esses achados coincidem com a literatura, sendo frisada a necessidade 
de atenção para as condições de saúde bucal, a fim de manter a conservação dentária adequada, proporcionando melhor qualidade de vida para essa população. (CARNEIRO et al., 2005; LIMA et al., 2009).

Embora o presente estudo tenha relatado oito $(53,33 \%)$ idosos utilizando prótese dentária, não existe associação significativa para o seu uso ou a sua ausência em relação aos aspectos avaliados da mastigação. O estudo de Fazito et al. (2004) afirma que o uso da prótese dentária melhora a capacidade de mastigação. Segundo Campos, Monteiro e Ornelas (2000), porém, a ausência de dentes e/ou o uso de próteses mal adaptadas podem levar o idoso à perda do apetite, devido à dificuldade para mastigar.

No estudo de Rocha e Lima (2010), a maioria dos idosos pesquisados apresentou alteração de tonicidade das estruturas orofaciais, considerando que há diminuição de massa e força muscular com o aumento da idade. Todavia, essas alterações não foram observadas neste estudo, concluindo-se que, mesmo com o envelhecimento, esses padrões estão próximos à normalidade. Desse modo, nota-se que há diferenças individuais no efeito desse processo, pois ele ocorre em tempos diferentes e não afeta todos os órgãos de forma padronizada, uma vez que é necessária a união de aspectos como o estilo de vida do sujeito, a genética que o afeta e o ambiente onde ele vive. (CANTERJI; CASTRO, 2002).

Em relação à avaliação de morfologia dos lábios, foram observados 2 (13,33\%) idosos com lábio superior fino, concordando com os achados do trabalho de Lima et al. (2009), cujo resultado da amostra com lábio superior fino foi de $8,8 \%$. Essa característica pode ser atribuída à atrofia muscular resultante do envelhecimento natural das estruturas do sistema estomatognático. (COUTO, 2002).

Em relação aos movimentos de mandíbula durante a alimentação, observou-se que seis (40\%) dos idosos apresentaram movimentos de mandíbula verticais, resultado semelhante ao encontrado por Lima et al. (2009), cujo trabalho apresentou 58,8\% da amostra com esse padrão. Vale ressaltar que dos seis idosos que apresentaram padrão de mastigação vertical, 5 relataram queixa de sempre ter dificuldade para mastigar os alimentos, convergindo com os achados de Lima et al. (2009), que observaram 35,5\% da amostra com a mesma queixa.

A velocidade lenta na mastigação foi encontrada em 12 idosos, dos quais 7 revelaram sempre ter dificuldade de mastigar. O mesmo padrão de velocidade durante o processo de mastigação foi encontrado em $50 \%$ dos idosos que participaram do estudo de Alencar, Pereira e Cysne (2003), o que leva a concluir que os sujeitos apresentam algum tipo de dificuldade no processo de mastigação devido a modificações na anatomia e morfologia resultantes do envelhecimento.

As características encontradas na avaliação da função estomatognática, tais como a lentidão, a participação da musculatura perioral e os movimentos verticais de mandíbula, não podem ser consideradas como mastigação alterada. De acordo com a literatura, as alterações 
decorrentes da diminuição do tônus da musculatura envolvida na mastigação são provocadas pelo envelhecimento e dificultam tanto os movimentos mandibulares quanto os de lateralização do bolo alimentar durante o processo de alimentação. (PERLMAN; SCHULZE-DERIEUS, 1997; SUZUKI, 1997). Em relação às características de mastigação da amostra avaliada, observou-se que $6(40 \%)$ dos sujeitos apresentaram participação exagerada da musculatura perioral, o que confronta os $82,4 \%$ encontrados no estudo de Lima et al. (2009).

A análise dos resultados deste estudo aponta diferença significativa para a idade desses idosos apenas se comparada à deglutição. Verificou-se que os idosos com a deglutição alterada apresentam idade significativamente superior à dos idosos com deglutição normal $(\mathrm{p}=0,006)$. Lima (2004) concluiu, em pesquisa realizada com idosos saudáveis, que a presença das alterações na deglutição nessa população pode ser causada pelo processo de envelhecimento em si. De acordo com Marcolino et al. (2009), as modificações/ alterações ocorridas na deglutição dos indivíduos saudáveis que se encontram na fase do envelhecimento é denominada "presbifagia". Observou-se que não houve associação significativa entre essas modificações e a queixa de mastigar e de deglutir os alimentos. Dessa forma, há indícios de que os idosos adaptam-se a essas modificações estruturais, a fim de obterem uma alimentação com maior eficiência.

\section{Conclusão}

As alterações mastigatórias predominantemente encontradas na amostra estudada não interviram, sobremaneira, no desempenho do processo de mastigação e deglutição dos idosos avaliados, mesmo com as queixas apresentadas. Esse fato pode estar relacionado às adaptações individuais realizadas durante essas funções, a fim de se manter a alimentação mais próxima do normal.

Orofacial myofunctional changes associated with aging process in a group institutionalized elderly

\section{Abstract}

With the aging population, health professionals must be prepared to work with this age group, since structural and physiological changes occur as a result of aging. To assess the consequences that the cause orofacial myofunctional alterations in the stomatognathic functions of chewing and swallowing in institutionalized older people. The study was conducted in Port-Society Assistance to Needy Alegrense, which evaluated 15 older people patients between 64 and 93 years, who complained to chew and/ or swallowing. An interview about the possible difficulties in the process of feeding. After protocols were applied for evaluation of stomatognathic structures and functions of chewing and swallowing. We found that $53,33 \%$ of older respondents make use of dental prosthesis. With regard to chewing, $40 \%$ had bilateral alternating chewing, 26,67\% right unilateral mastication and $40 \%$ exaggerated chewing with participation of the perioral muscles. Chew slowly was observed in $86,67 \%$ of the measured and vertical movement of the jaw in $40 \%$. Also, there was swallowing with changes in $26.67 \%$ 
of the older people, of which $13,33 \%$ were choking when swallowing, $6,67 \%$ and a cough $6,67 \%$ showed noisy swallowing. No significant difference was found between changes in chewing and swallowing, and performance in these processes, even with abuse in performing the functions and structures change in stomatognathic.

Keywords: Aging. Health of the elderly. Chew. Dental prosthesis. Stomatognathic system.

\section{Referências}

ALENCAR, S. C.; PEREIRAS. M. S.; CYSNE C. O processo de envelhecimento e as características da função mastigatória. Revista CEFAC Saúde e Educação, São Paulo, v. 3, n. 5, p. 247-250, 2003.

ALTMANN, E. B. C. Deglutição atípica. In: KUDO, M. (Coord.). Fisioterapia, fonoaudiologia e terapia ocupacional em pediatria. São Paulo: Sarvier, 1990. p. 116-131.

BIANCHINI, E. M. G. A cefalometria nas alterações miofuncionais: diagnóstico e tratamento fonoaudiológico. 5. ed. Carapicuíba, SP: Pró-Fono, 2002.

BIAZUZ, M.; BALBINOT, N.; WIBELINGER, L. M. Avaliação do risco de quedas em idosos. Revista Brasileira de Ciências do Envelhecimento Humano, Passo Fundo, v. 7, n. 1, p. 34-41, jan./abr. 2010.

BOURDIOL, P.; MIOCHE, L. Correlations between functional and occlusal tooth-surface areas and food texture during natural chewing sequences in humans. Archives of Oral Biology, v. 45, n. 8, p. 691-699, 2000.

CAMPOS, M. T. F. S.; MONTEIRO, J. B. R.; ORNELAS, A. P. R. C. Fatores que afetam o consumo alimentar e a nutrição do idoso. Revista de Nutrição, Campinas, v. 13, n. 3, p. 157-165, dez. 2000.

CANTERJI, M. B.; CASTRO S. A. F. N. Idoso, vida cotidiana e participação social. In: TERRA, N. L.; DORNELLLES, B. (Org.).
Envelhecimento bem-sucedido. 2. ed. Porto Alegre: Edipucrs, 2002. p. 271-281.

CANTERJI, M. B. et al. Avaliação de funções estomatognáticas em idosos institucionalizados e independentes em uma instituição geriátrica de Porto Alegre. Scientia Medica, Porto Alegre, v. 14, n. 1, p. 59-66, jan./mar. 2004.

CARNEIRO, R. M. V. et al. Saúde bucal de idosos institucionalizados, zona leste de São Paulo. Caderno Saúde Pública, Rio de Janeiro, v. 21, n. 6, p. 1709-1716, 2005.

CHAIMOWICZ, F.; GRECO, D. B. Dinâmica da institucionalização de idosos em Belo Horizonte, Brasil. Revista Saúde Pública, São Paulo, v. 33, n. 5, p. 454-460, 1999.

COUTO, E. A. B. Avaliação pelo fonoaudiólogo. In: MACIEL, A. (Org.). Avaliação multidisciplinar do paciente geriátrico. Rio de Janeiro: Revinter, 2002. p. 157-177.

DAVIM, R. M. B. et al. Estudo com idosos de instituições asilares no município de Natal/RN: características socioeconômicas e de saúde. Revista Latino-Americana de Enfermagem, Ribeirão Preto, v. 12, n. 3, p. 518-524, 2004.

DIAS, B. K. P.; CARDOSO, M. C. A. F. Características da função de deglutição em um grupo de idosas institucionalizadas. Estudo Interdisciplinar do Envelhecimento, Porto Alegre, v. 14, n. 1, p. 107-124, 2009.

FAZITO, L. T. et al. Comparação das queixas alimentares de idosos com e sem prótese dentária. Revista CEFAC Saúde e Educação, São Paulo, v. 6, n. 2, p. 143-150, abr./jun. 2004.

FREITAS JUNIOR, A. C. et al. Envelhecimento do aparelho estomatognático: alterações fisiológicas e anatômicas. Revista Odontológica de Araçatuba, Araçatuba, v. 29 , n. 1 , p. $47-52,2008$.

GUEDES, J. M.; SILVEIRA, R. C. Análise da capacidade funcional da população geriátrica institucionalizada na cidade de Passo Fundo - RS. Revista Brasileira de Ciências do Envelhecimento Humano, Passo Fundo, v. 1, n. 2, p. 10-21, 2004. 
GUTIERREZ, S. M. et al. Queixas fonoaudiológicas de idosos residentes em uma instituição de longa permanência. Revista Distúrbio da Comunicação, São Paulo, v. 21, n. 1, p. 21-27. 2009.

INSTITUTO BRASILEIRO DE GEOGRAFIA E ESTATÍSTICA(IBGE). Censo demográfico 2010. Rio de Janeiro: IBGE, 2010.

LIMA, L. K. Análise das fases oral e faríngea da deglutição em idosos independentes e institucionalizados. 2004. 114 f. Dissertação (Mestrado em Gerontologia Biomédica) - Instituto de Geriatria e Gerontologia, Pontifícia Universidade Católica de São Paulo, Porto Alegre, 2004.

LIMA, R. M. F. et al. Adaptações na mastigação, deglutição e fonoarticulação em idosos de instituição de longa permanência. Revista CEFAC Saúde e Educação, São Paulo, v. 11, supl. 3, p. 405-422, 2009.

LOCKER, D. et al. Oral health-related quality of life of a population of medically compromised elderly people. Community Dent Health, Worral, v. 19, n. 2, p. 90-97, 2002.

KOSSIONI, A. E.; DONTAS, A. S. The stomatognathic system in the elderly. Useful information for the medical practitioner. Clinical Interventions in Aging, United Kingdom, v. 2, n. 4, p. 591-597, 2007.

MOJON, P.; BUDTZ-JORGENSEN, E.; RAPIN, C. H. Relationship between oral health and nutritition in very old people. Age and Age, v. 28, n. 5, p. 465-473, 1999.

MARCHESAN, I. Q. Avaliando e tratando o sistema estomatognático. In: LOPES-FILHO, O. (Ed.). Tratado de fonoaudiologia. São Paulo: Roca, 1997. p. 763-780.

Protocolo de avaliação miofuncional orofacial. In: KRAKAUER H. L.; FRANCESCO R.; MARCHESAN, I. Q. (Org.). Respiração oral. São José dos Campos, SP: Pulso, 2003. p. 55-79.

MARCOLINO, J. et al. Achados fonoaudiológicos na deglutição de idosos do município de Irati - Paraná. Revista Brasileira de
Geriatria e Gerontologia, Paraná, v. 12, n. 2, p. 193-200, 2009.

MENDES, F. S.; TCHAKMAKIAN, L. A. Qualidade de vida e interdisciplinaridade: a necessidade do programa de assistência domiciliar na prevenção das complicações em idosos com disfagia. O Mundo da Saúde, São Paulo, v. 33, n. 3, p. 320-328, 2009.

O’LOUGHLIN, G.; SHANLEY, C. Swallowing problems in the nursing home: A novel training response. Dysphagia, Summer, v. 13, n. 3, p. 172-183, 1998.

PERLMAN, A. L.; SCHULZE-DERIEUS, K. S. Deglutition and its disorders: anatomy, physiology, clinical diagnosis, and management. San Diego: Singular Publishing Group, 1997.

ROCHA, M. A. S; LIMA, M. L. Caracterização dos distúrbios miofuncionais orofaciais de idosos institucionalizados. Revista Geriatria \& Gerontologia, Rio de Janeiro, v. 4, n. 1, p. 21-26, 2010.

SASAHARA, A. E. A.; LIMA, E. M. Atuação da fonoaudiologia hospitalar na disfagia. In: PAPALÉO NETTO, M.; BRITO, F. C. O. (Org.). Urgências em geriatria: epidemiologia, fisiologia, quadro clínico, conduta terapêutica. São Paulo: Atheneu, 2001. p. 53-68.

SILVA, L. G.; GOLDENBERG, M. A mastigação no processo de envelhecimento. Revista CEFAC Saúde e Educação, São Paulo, v. 3, n. 1, p. 27-35, 2001.

SUZUKI, H. S. O entardecer da deglutição: um estudo sobre modificações nos hábitos da rotina alimentar x fisiologia da deglutição do idoso normal. 1997. 33f. Monografia (Especialização em Motricidade Oral) - Pós-Graduação em Saúde e Educação, CEFAC, São Paulo, 1997. 